\title{
Early cranial ultrasound findings among infants with neonatal encephalopathy in Uganda: an observational study
}

\author{
Cally J. Tann ${ }^{1-3}$, Margaret Nakakeeto ${ }^{4}$, Cornelia Hagmann ${ }^{5}$, Emily L. Webb ${ }^{2}$, Natasha Nyombi ${ }^{6}$, Flaviah Namiiro ${ }^{6}$, \\ Kelly Harvey-Jones ${ }^{1}$, Anita Muhumuza ${ }^{6}$, Kathy Burgoine ${ }^{1}$, Alison M. Elliott ${ }^{2,3}$, Jennifer J. Kurinczuk ${ }^{7}$, Nicola J. Robertson ${ }^{1}$ \\ and Frances M. Cowan ${ }^{8}$
}

BACKGROUND: In sub-Saharan Africa, the timing and nature of brain injury and their relation to mortality in neonatal encephalopathy (NE) are unknown. We evaluated cranial ultrasound (cUS) scans from term Ugandan infants with and without NE for evidence of brain injury.

METHODS: Infants were recruited from a national referral hospital in Kampala. Cases (184) had NE and controls (100) were systematically selected unaffected term infants. All had cUS scans $<36 \mathrm{~h}$ reported blind to NE status.

RESULTS: Scans were performed at median age 11.5 (interquartile range (IQR): 5.2-20.2) and 8.4 (IQR: 3.6-13.5) hours, in cases and controls respectively. None had established antepartum injury. Major evolving injury was reported in $21.2 \%$ of the cases vs. $1.0 \%$ controls $(P<0.001)$. White matter injury was not significantly associated with bacteremia in encephalopathic infants (odds ratios (OR): 3.06 (95\% confidence interval (CI): 0.98-9.60). Major cUS abnormality significantly increased the risk of neonatal death (case fatality 53.9\% with brain injury vs. 25.9\% without; OR: 3.34 (95\% Cl: $1.61-6.95)$ ).

CONCLUSION: In this low-resource setting, there was no evidence of established antepartum insult, but a high proportion of encephalopathic infants had evidence of major recent and evolving brain injury on early cUS imaging, suggesting prolonged or severe acute exposure to hypoxia-ischemia (HI). Early abnormalities were a significant predictor of death.

$\mathbf{N}$ eonatal encephalopathy (NE) is a leading cause of neonatal mortality and morbidity worldwide affecting more than one million infants around the world each year $(1,2)$. There is a substantial body of evidence from high-income settings supporting an injury pattern on neuroimaging consistent with hypoxia-ischemia (HI) that occurs in the immediate perinatal period (3-5). However, the nature and timing of brain injury leading to $\mathrm{NE}$ in the term infant is poorly understood, particularly in low-income settings. NE may be considered a multifactorial disease involving a complex causal pathway with the contribution of individual risk factors likely to change according to their prevalence, frequency of exposure, care setting, and definition of NE used (6). In low-income countries, difficulties in accurate fetal monitoring and lack of standardized recording of intrapartum complications, mean that measures of intrapartum-related hypoxia, including cause and duration, are difficult to define (1). Delays in identification and referral of women experiencing obstetric and fetal complications may have important effects on the nature and timing of $\mathrm{HI}$ insults (7). Preclinical study findings suggest that newborn infection may sensitize the immature term brain to subsequent injury $(8,9)$, a potentially important factor in low-resource settings.

Understanding the nature, timing of onset, and duration of $\mathrm{HI}$ are key to evaluating which neuroprotective strategies may be effective (10). In high-income countries (HICs), magnetic resonance (MR) imaging, including diffusion-weighted imaging and MR spectroscopy, has been used to identify the nature and timing of insults and to predict neurodevelopmental outcome (4,11-13), however, MR imaging is not available in the majority of low-income countries. A decline in brain high energy phosphates seen on MR spectroscopy over the first 8-24h after birth in babies with NE (14), a phenomenon called secondary energy failure, has been observed to occur typically in parallel with the cascade of programmed cell death following a HI insult (15). In keeping with this evolving injury, abnormalities seen on cranial ultrasound (cUS) imaging, such as central gray matter, white matter, and cortical echodensities indicative of neuronal death, are frequently not evident on cUS or MR imaging until $24-48 \mathrm{~h}$ after HI $(13,16)$.

The aims of this study were: (i) to evaluate cUS scans obtained soon after birth from term Ugandan infants with NE and unaffected term control infants for evidence of established and evolving brain injury, and (ii) to assess the contribution of perinatal infection to the pattern of brain injury.

\footnotetext{
IInstitute for Women's Health, University College London, London, UK; ${ }^{2}$ London School of Hygiene \& Tropical Medicine, London, UK; ${ }^{3}$ MRC/UVRI Uganda Research Unit on AIDS, Entebbe, Uganda; ${ }^{4}$ Uganda Women's Health Initiative, Kampala, Uganda; ${ }^{5}$ Department of Neonatology, University Hospital Zurich, Zurich, Switzerland; ${ }^{6}$ Department of Paediatrics, Mulago Hospital, Kampala, Uganda; ${ }^{7}$ National Perinatal Epidemiology Unit, University of Oxford, Oxford, UK; ${ }^{8}$ Department of Paediatrics, Imperial College London, London, UK. Correspondence: Cally J.Tann (cally.tann@lshtm.ac.uk); c.tann@uclh.nhs.uk

Received 26 October 2015; accepted 2 February 2016; advance online publication 25 May 2016. doi:10.1038/pr.2016.77
} 


\section{RESULTS}

\section{Study Participants}

In total, early cUS was performed and assessed for 184 (87.6\%) of 210 case infants. Among the 409 ABAaNA controls infants, 105 infants were recruited sequentially from the start of the study as long as there was a study doctor available to perform the cUS. Of these, 69 were recruited from high-risk labor ward, and 36 recruited from the low-risk labor ward. After exclusion for poor image quality, 100 control infants remained (Figure 1). No significant differences were seen in baseline demographic and clinical characteristics between those with and without early scans, in either cohort (data not shown). Good-quality images from the second scan (day 4/5) were available for 88 surviving infants with encephalopathy (Figure 1).

Baseline demographic and clinical characteristics of the cohorts are shown in Table 1. The majority of encephalopathic infants were moderately or severely affected (89.7\%) (Table 1). A third of case infants died in the neonatal period and half had clinical seizures.

\section{Comparison Between Cases and Controls}

Median age at scanning was $11.5 \mathrm{~h}$ (interquartile range (IQR): 5.2-20.2 h), and 8.4h (IQR: $3.6-13.5 \mathrm{~h}$ ) in cases and controls, respectively $(P=0.0019)$. The distribution of basal gangliathalami (BGT) and white matter (WM) scores among case and control infants is shown in Table 2.

No abnormality was found in $45.1 \%(83 / 184)$ of cases and $67.0 \%(67 / 100)$ controls $(P<0.001)$. Mild WM changes and mild/unilateral echogenicity in the BGT (score 1 and not indicative of major abnormality) were common among cases and controls. More case than control infants had mild WM changes (WM score 1, odds ratios (OR): 3.00 (95\% confidence interval (CI): 1.46-6.15). There was no significant difference in mild BGT scores (BGT score 1, OR: 1.65 (95\% CI: 0.96-2.85). One control had a score of 2 for BGT echogenicity (Table 2); this scan was reviewed postanalysis (by F.M.C.) and the score was considered marginal between 1 and 2 and the WM and cortex found normal. Score 3 for WM or BGT was uncommon (Table 2) among cases and absent among controls.

Overall, scans obtained within $36 \mathrm{~h}$ of birth showed a major abnormality in one fifth of encephalopathic infants (Table 3 ). When the analysis was restricted to scans obtained within $18 \mathrm{~h}$ of birth, the high case prevalence of major abnormalities persisted (17.5\%) (Table 3).

\section{Pattern and Timing of Injury in NE}

The pattern of injury seen among infants with NE is shown in Table 4. All major abnormalities (score $\geq 2$ ) were of recent onset: no established injury with tissue atrophy, parenchymal cystic change, definite developmental abnormality, or large parenchymal hemorrhage was seen in either cases or controls. Subependymal pseudocysts, caudothalamic cysts, and choroid plexus cysts were common among all infants $(12.5 \%(23 / 184)$ in cases vs. $17 \%$ (17/100) in controls).

Serial scans were performed on day 4/5 in 88 NE infants: 74 had normal early scans, 35 (47.3\%) becoming abnormal by day 4/5. In the 14 with abnormal early cUS, 5 normalized and 9 remained abnormal.

\section{Neonatal Infection and Brain Injury}

Among cases, bacteremia was detected in $20.0 \%(5 / 25)$ of infants with evidence of WM injury vs. $7.6 \%(12 / 159)$ of those without (OR: 3.06 (95\% CI: 0.98-9.60) and in 18.2\% (4/22) of cases with major BGT abnormality vs. $8.0 \%$ (13/162) of those without (OR: 2.55 (95\% CI: 0.75-8.65)).

\section{Neonatal Case Fatality}

Among encephalopathic infants, a major abnormality seen on early cUS $(<36 \mathrm{~h})$ tripled the odds of neonatal death (case fatality with major abnormality $53.9 \%(21 / 39)$ vs. $25.9 \%(37 / 143)$ with no major abnormality (OR: 3.34 (95\% CI: 1.61-6.95)). A similar pattern of association was seen for those scanned
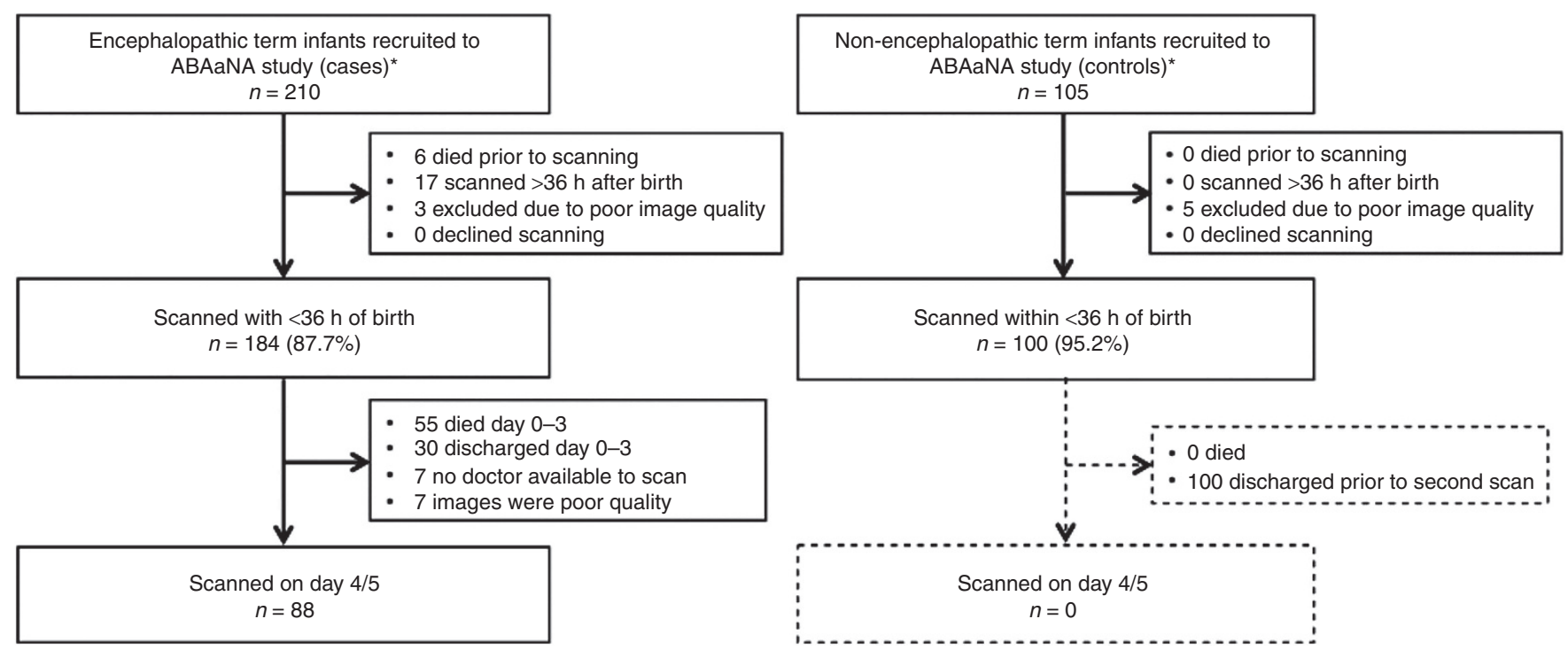

Figure 1. Flow diagram of study participants. 


\section{Articles $\mid$ Tann etal.}

Table 1. Baseline demographic and clinical characteristics of mothers and babies

\begin{tabular}{|c|c|c|c|}
\hline & Case $N(\%)$ & Control N (\%) & $P$ value $^{b}$ \\
\hline \multicolumn{4}{|l|}{ Maternal } \\
\hline $\begin{array}{l}\text { Maternal education } \\
\leq \text { primary level }\end{array}$ & $72 / 184(39.1)$ & $39 / 100(39.0)$ & 0.983 \\
\hline Primiparity & $112 / 184(60.9)$ & $48 / 100(48.0)$ & 0.037 \\
\hline $\begin{array}{l}\text { Emergency } \\
\text { cesarean section }\end{array}$ & $43 / 183(23.5)$ & $16 / 100(16.0)$ & 0.138 \\
\hline \multicolumn{4}{|l|}{ Neonatal } \\
\hline Male sex & $116 / 184(63.0)$ & $51 / 100(51.0)$ & 0.049 \\
\hline $\begin{array}{l}\text { Mean birth } \\
\text { weight (g) (range) }\end{array}$ & $3,196(1,940-4,640)$ & $3,065(1,640-4,290)$ & 0.023 \\
\hline 1-min Apgar $<3$ & $60 / 176(34.1)$ & $1 / 100(1.0)$ & $<0.001$ \\
\hline 5-min Apgar $<7$ & $118 / 162(72.8)$ & $1 / 100(1.0)$ & $<0.001$ \\
\hline \multicolumn{4}{|c|}{ Clinical features of encephalopathic infants } \\
\hline \multicolumn{4}{|c|}{ Grade of encephalopathy ${ }^{a}$} \\
\hline Mild & $19 / 184(10.3)$ & - & \\
\hline Moderate & $105 / 184(57.1)$ & - & \\
\hline Severe & $60 / 184(32.6)$ & - & \\
\hline Absent suck & $145 / 183(79.2)$ & - & \\
\hline Clinical seizures & $100 / 184(54.4)$ & - & \\
\hline Comatose & $48 / 184(26.1)$ & - & \\
\hline Neonatal case fatality & $58 / 182(31.9)$ & - & \\
\hline
\end{tabular}

aGrade encephalopathy defined according to Sarnat and Sarnat classification (18) on

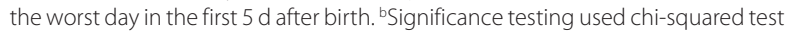
except for mean birth weight where $t$-test for comparison of means was used.

Table 2. Distribution of basal ganglia-thalami and white matter scores for case and controls

\begin{tabular}{lcc}
\hline & Cases $(n=184) n(\%)$ & Controls $(n=100) n(\%)$ \\
\hline BGT score & $100(54.4)$ & $72(72.0)$ \\
0 & $62(33.7)$ & $27(27.0)$ \\
1 & $19(10.3)$ & $1(1.0)$ \\
2 & $3(1.6)$ & $0(0.0)$ \\
3 & & \\
WM score & $116(63.0)$ & $89(89.0)$ \\
0 & $43(23.4)$ & $11(11.0)$ \\
1 & $24(13.0)$ & $0(0.0)$ \\
2 & $1(0.5)$ & $0(0.0)$ \\
3 & & \\
\hline
\end{tabular}

$\mathrm{BGT}$, basal ganglia-thalami; WM, white matter.

within 18 h of birth (case fatality $59.1 \%(13 / 22)$ vs. $29.1 \%$ (30/103), respectively (OR: 3.51 (95\% CI: 1.36-9.10)).

\section{DISCUSSION}

Our study showed a high prevalence of recent, evolving brain injury among encephalopathic infants on early cUS imaging, in this low-resource Ugandan setting. Evidence of established injury, indicative of an antepartum insult or congenital
Table 3. Proportion of infants with major abnormalities on early cUS imaging

\begin{tabular}{|c|c|c|c|c|}
\hline Age at imaging & Case N (\%) & Control $N(\%)$ & OR & $95 \% \mathrm{Cl}$ \\
\hline cUS performed $<36 \mathrm{~h}$ & $39 / 184(21.2)$ & $1 / 100(1.0)$ & 26.6 & $3.60-197.01$ \\
\hline cUS perfomed $<18 \mathrm{~h}^{\mathrm{a}}$ & $22 / 126(17.5)$ & $1 / 85(1.2)$ & 17.77 & $2.35-134.56$ \\
\hline
\end{tabular}

Table 4. Pattern of major abnormality seen on early cUS among encephalopathic (case) infants $(N=184)$

\begin{tabular}{lc}
\hline Major abnormality & $N(\%)$ \\
\hline No abnormality & $145(78.8 \%)$ \\
BGT abnormality & $22(12.0 \%)$ \\
WM abnormality & $25(13.6 \%)$ \\
Global abnormality & $8(4.4 \%)$ \\
\hline a'Major abnormality in BGT or WM defined as a score of $\geq 2$ in that area (injury seen in \\
BGT and WM are not mutually exclusive). ${ }^{b} G$ lobal abnormality was defined as a score $\geq 2$ \\
in both the BGT and WM \\
BGT, basal ganglia-thalami; CUS, cranial ultrasound; WM, white matter
\end{tabular}

pathology, was not seen. Signs of major evolving injury, suggestive of a relatively recent as opposed to an established antepartum brain insult, affected one in five encephalopathic infants. In infants with encephalopathy, the presence of a major evolving brain injury tripled the likelihood of dying in the neonatal period. There was no significant increase in white matter injury seen among encephalopathic infants with neonatal infection.

Benefits of cUS are that it can be performed serially, at the bedside, and in the sickest of infants (17). It allows the early detection of congenital or another non-HI cause of NE and informs the timing of $\mathrm{HI}$ injury; as a consequence, it continues to be recommended as an important adjunct to MRI scanning for encephalopathic infants with NE in HICs $(17,18)$. Previous studies from HICs have found abnormal cUS to be predictive of outcome $(3,17)$, with good correlation between MRI and cUS findings among infants with HIE in some, but not all studies $(3,19)$.

The cUS scoring system used by us successfully identified encephalopathic infants with major abnormalities on cUS, and differentiated between abnormalities suggestive of significant brain injury and other changes frequently seen among well term infants (20). Studies from HICs have shown unilateral BGT changes, equivalent to our score 1 , to be associated with a more benign prognosis $(3,21)$, suggesting that they are poor predictors of adverse neurodevelopmental outcome. This is likely to extend to our Ugandan population where focal unilateral central gray matter changes and focal WM echogenicity were found to be common among well term Ugandan newborns (22).

This is the first study in a sub-Saharan low-income setting to use cUS to examine the nature and timing of newborn brain injury. A study from South India examined perinatal MRI findings among encephalopathic infants (23). That 
study reported minimal antepartum established injury on MRI performed within $3 \mathrm{wk}$ of birth. White matter injury was also common in this study (in 91\%) and around a quarter had deep gray matter injury (23). Comparison of our results with other cUS studies from HICs is hampered by differing definitions of NE used and inconsistency of brain abnormality definition. In the Netherlands, early changes on cUS ( $<6 \mathrm{~h}$ of age) were not found to be predictive of outcome, with most infants with severe encephalopathy developing cUS abnormalities between $24-72 \mathrm{~h}$ of age (19). A good correlation was seen between later cUS abnormalities and histological findings (16).
Defining the nature and timing of newborn brain injury in $\mathrm{NE}$ is key to understanding the most appropriate prevention and intervention strategies for NE. In this study, one in five encephalopathic infants had features of evolving brain injury on very early (median $11 \mathrm{~h}$ ) scans. Since changes seen on cUS are recognized to take some time to develop after the original brain insult (19), this may imply that the injury pathway begins several hours before delivery, or a very severe acute exposure to HI. It is unclear whether severe injury may appear on cUS more rapidly than moderate injury; experimental studies show that secondary energy failure develops more rapidly in severe $\mathrm{HI}$ than moderate HI (24). The accelerated cell death cascade

Table 5. Scoring system used for basal ganglia and white matter abnormalities

\begin{tabular}{lcll}
\hline & Score & Basal ganglia and thalami & White matter \\
\hline No abnormality & 0 & No abnormality & No abnormality \\
Minor abnormality & 1 & Mild_swollen and/or small focal unilateral abnormality & Mildly echogenic or patchy \\
Major abnormality & 2 & Clearly demarcated focal bilateral echogencity & More diffuse and moderate/severe echogenicity \\
& 3 & Bilateral, severe, extensive abnormalities & $\begin{array}{l}\text { Dense echogenicity and/or parenchymal cysts, atrophy, } \\
\text { organizing hemorrhage }\end{array}$ \\
\hline
\end{tabular}

a

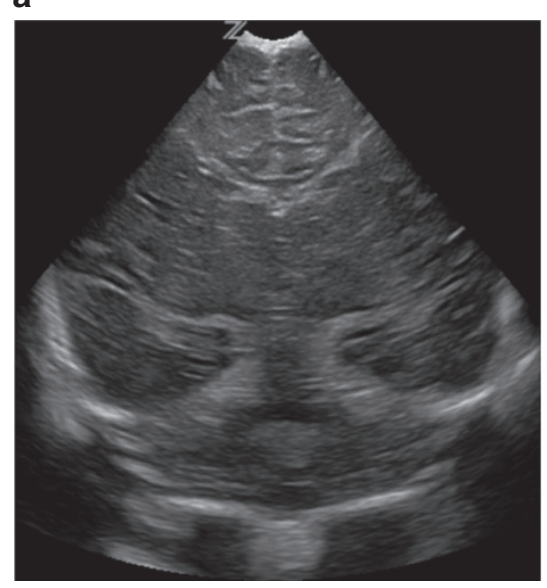

b

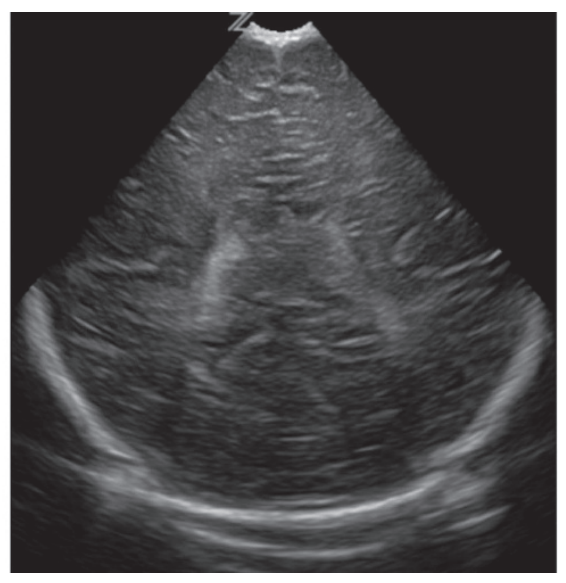

c

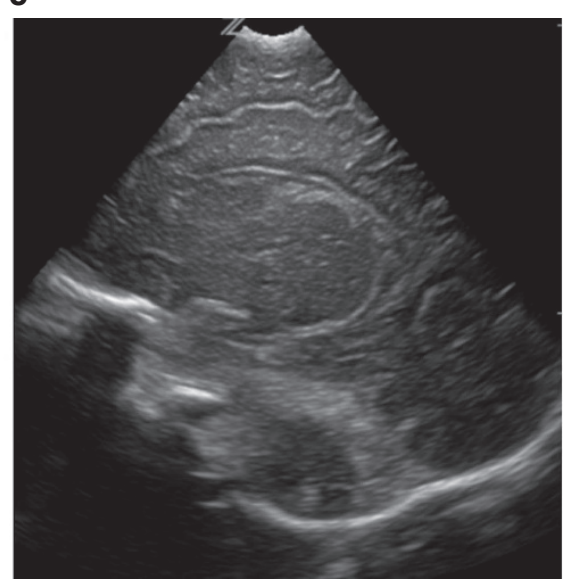

Figure 2. Normal basal ganglia and white matter seen on coronal $(\mathbf{a}, \mathbf{b})$ and parasagittal $(\mathbf{c})$ images consistent with a score of 0 .

a

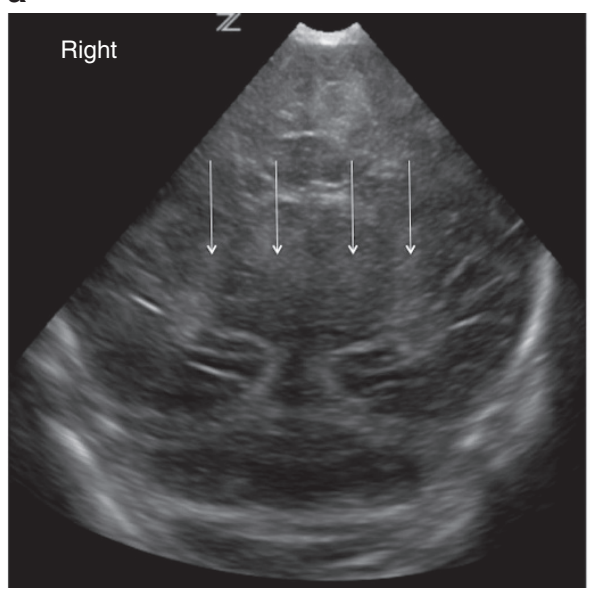

\section{b}

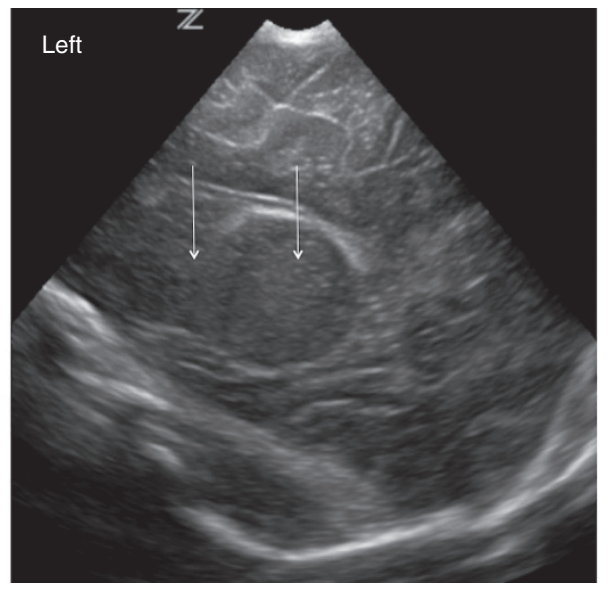

Figure 3. Bilateral basal ganglia and thalamic echogenicity seen on coronal (a) and parasagittal (b) images consistent with a score of 2. 


\section{Articles | Tannetal.}

may be reflected in earlier evidence of injury on cUS. As in South India, a high prevalence of WM injury was seen among cases on early imaging. This may be associated with perinatal infection and inflammation or may reflect other exposures, including a less acute and more chronic HI insult. MRI diagnostic techniques could have contributed substantially to defining the nature and timing of brain injury $(13,23,25)$, but unfortunately are not currently available in Uganda.

This study has limitations and challenges. First, all selection, interobserver and recall bias cannot be excluded completely. The case cohort is likely to have under-represented infants at either end of the spectrum of NE severity since the most severely affected often died prior to recruitment, and mildly affected infants may go undetected. Infants born on the lowrisk labor ward were slightly over-represented in the control group and cases were a median of $3 \mathrm{~h}$ older than controls when scanned. However, it seems unlikely that any of these factors contributed substantially to the large differences seen between case and control infants. Scans were read by two external experts blind to study status and all clinical and demographic data; so, minimizing observer bias but all inter- and intraobserver bias cannot be excluded. Finally, since participants were recruited from a high-risk urban Ugandan referral centre, they may not reflect findings among infants from other, more rural populations.

\section{Conclusions}

In this study, in a Ugandan hospital setting, there was no cUS evidence of established antepartum injury, but a fifth of encephalopathic infants had evidence of major recent and evolving brain injury on early cUS imaging performed at a median age of $11 \mathrm{~h}$. Since we assume from studies in HICs that it takes $>24 \mathrm{~h}$ for injury to become apparent on cUS, this may suggest a brain insult occurring several hours before delivery or a very severe acute exposure to HI. These early abnormalities were significant predictors of death in this population.

\section{METHODS}

This was a nested study of early brain imaging, conducted within a larger case-control study designed to investigate the etiology of NE in a

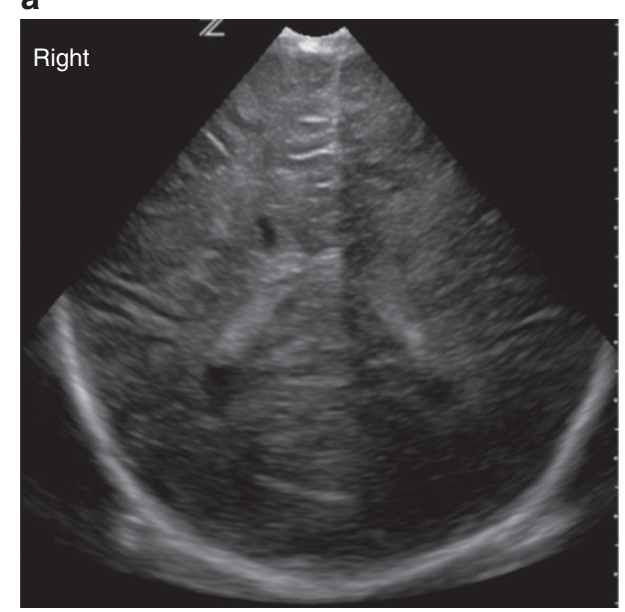

b

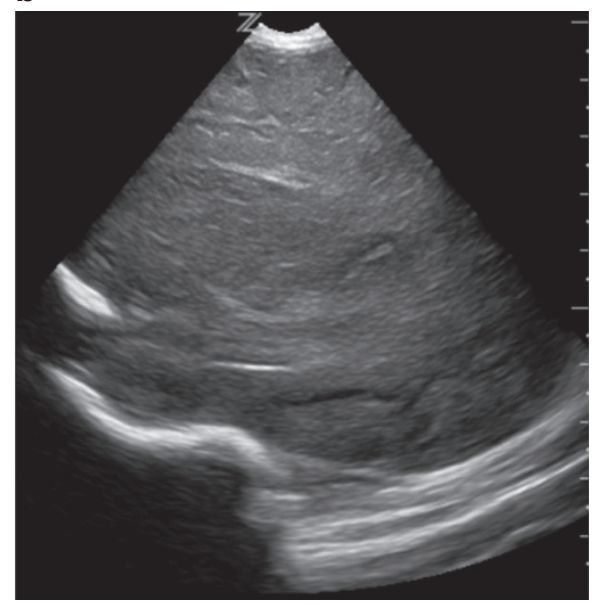

Figure 4. Diffuse, moderate/severe echogenicity in the white matter seen on coronal (a) and parasagittal (b) images consistent with a score of 2.

a

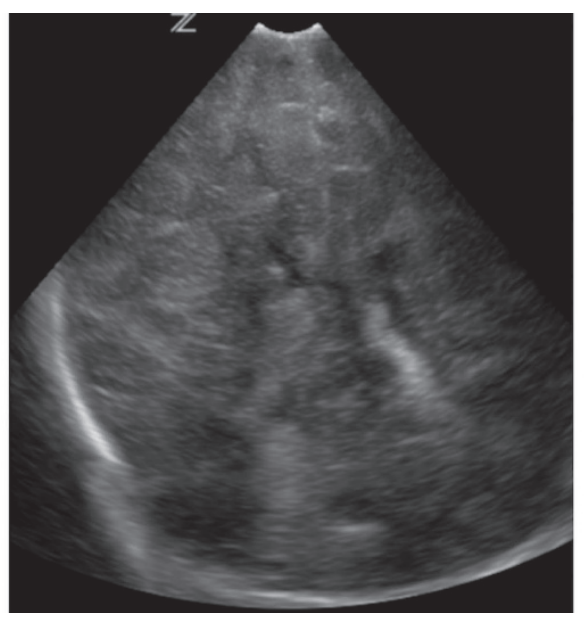

b

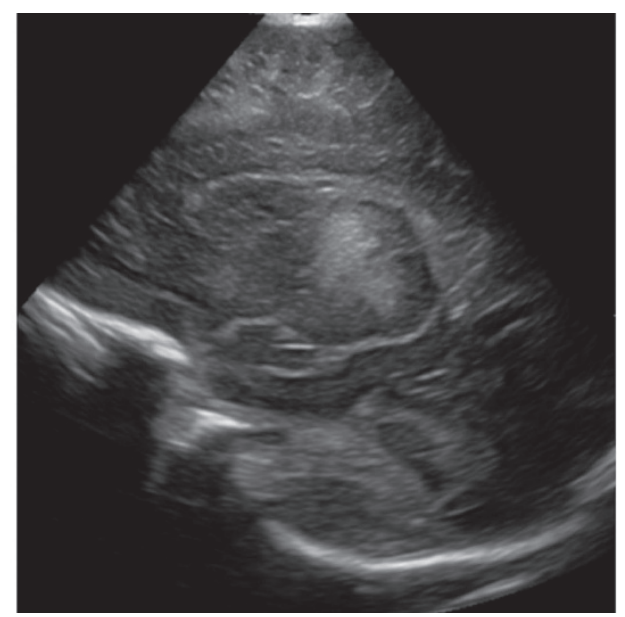

Figure 5. Severe dense echogenicity in the basal ganglia and thalami on parasagittal view (a) and global echogenicity and swelling in the white matter and cortex on coronal images (b) consistent with a score of 3. 
Uganda (Associations between Birth Asphyxia and infections among Newborns in Africa-the "ABAaNA study"). Between 11 September 2011 and 3 October 2012, 210 case and 409 control infants were recruited to the main study. The study was approved by the ethics committees of the London School of Hygiene and Tropical Medicine, Uganda Virus Research Institute and Mulago Hospital, and the Uganda National Council for Science and Technology

\section{Setting}

All infants were born and recruited at Mulago Hospital, a National Referral Hospital in Kampala, Uganda's capital city. Over the 13-mo study period, 36,926 deliveries occurred at Mulago Hospital, 23\% $(8,767)$ by cesarean section. Women deliver on the low-risk or highrisk labor ward (ratio 1:4). Fetal monitoring uses Pinard stethoscope for intermittent auscultation. Neonatal resuscitation, performed by attending midwives, includes oxygen and bag-mask ventilation. Sick newborns are transferred to the Special Care Unit. Piped oxygen and simple continuous positive airway pressure support are available, but not intubation and ventilation. Mothers care for well infants on the postnatal wards.

\section{Study Participants}

Participants were term infants $<12 \mathrm{~h}$ of age with written parental informed consent, taken by trained study staff in the local language. NE case infants had a Thompson score of $\geq 6$ (ref. (26)). Sarnat \& Sarnat classification of encephalopathy severity (27) was assigned on the most severe day (between days 1-5). Contemporaneously recruited, unmatched controls (Thompson score $\leq 3$ ) were systematically sampled from the labor ward admissions. For this cUS substudy, a comparison group of 100 control infants were eligible for inclusion. Information was collected from antenatal and hospital clinical records and structured maternal interviews in the local language. Blood culture and species-specific bacterial real-time PCR assays detected neonatal bacteremia among all cases as previously described (28). Survival outcomes were recorded at $4 \mathrm{wk}$.

\section{Cranial Ultrasound Scans}

Scans were performed using a portable ultrasound machine (z.one ultra-Convertible Ultrasound System; Zonare Medical Systems, Mountain View, CA) with the P10-4 Phased Array Transducer (bandwidth 4-10 MHz with tissue and compound harmonics of $8 \mathrm{Hx}$ ).

Study doctors (K.B., K.H.-J., N.N., F.N., A.T.) were trained to perform cUS by CT and FC using methods described previously $(22,29,30)$, Minimally, five coronal, one midline sagittal and two parasagittal views on left and right were taken to visualize general anatomy, BGT, and WM. Scans were performed on recruitment (as soon as a trained study doctor was available) and again on day $4 / 5$ in all surviving infants that remained in-patients. Images were downloaded, anonymized, and reported from DICOM-formatted images (OsiriX software, Geneva, Switzerland) by F.M.C. and C.H., blind to case/control status and all clinical data. Abnormalities were scored according to a system adapted from van Wezel-Meijler et al., (17) and used by us previously in Uganda (Table 5) (22).

As previous cUS studies in HICs suggest that changes on neuroimaging become evident $>24 \mathrm{~h}$ after $\mathrm{HI}(13,16)$, only infants scanned for the first time within the first $36 \mathrm{~h}$ after birth were included. Our aim was to assess whether these scans appeared (i) normal (ii) had evidence of longstanding changes consistent with an antepartum insult or a developmental abnormality (parenchymal cysts, calcification, atrophy, organizing hemorrhage) or (iii) evidence of a recent event, typical of acute or subacute HI injury (extensive echogenicities in the BGT and WM). Signs of hemorrhage and focal arterial territory infarction were also looked for. Isolated cysts in the choroid plexus, subependymal cysts, cysts in the caudo-thalamic notch, and lenticulostriate vasculopathy were not considered as major abnormalities (19).

Examples of image findings and their relationship to scores are shown in Figures 2-5. The presence of a "major abnormality" was defined as a score of 2 or 3 in either BGT, WM, or both. Cortex abnormalities were not graded as it was difficult to obtain systematic views on all infants and cortical abnormalities are seldom seen in isolation of BGT and WM changes.

\section{Statistical Analysis}

Analysis was performed using Stata version 11.0 (StataCorp, College Station, TX). Chi-squared test, $t$-test, and Wilcoxon rank sum test were used to compare proportions, means, and medians (where data was not normally distributed), respectively. Univariable logistic regression analysis was used to calculate OR and 95\% CI. $P<0.05$ was taken as the formal level of statistical significance. Case fatality was defined as a death occurring in the neonatal period $(0-28 \mathrm{~d})$ as a proportion of infants at risk.

\section{ACKNOWLEDGMENTS}

The authors thank their parents, guardians, and children who participated in the study; the ABAaNA study team for their dedicated contribution to data collection and care of study participants; to Jamiir Mugalu, Jolly Nankunda, and all on the Special Care Unit at Mulago Hospital. The authors also thank the MRC/UVRI Uganda Research Unit on AIDS, the Uganda Women's Health Initiative and the Wellcome Bloomsbury Centre, LSHTM for their administrative support. We are grateful to Zonare Medical Systems for maintenance and upgrade of the z.one ultrasound system and UCL Institute for Women's Health for loan of the scanning equipment.

\section{STATEMENT OF FINANCIAL SUPPORT}

The study was funded by a Wellcome Trust Bloomsbury PhD Clinical Fellowship to C.J.T.

Disclosure: All authors have no financial relationships to disclose or conflicts to resolve.

\section{REFERENCES}

1. Lee AC, Kozuki $\mathrm{N}$, Blencowe $\mathrm{H}$, et al. Intrapartum-related neonatal encephalopathy incidence and impairment at regional and global levels for 2010 with trends from 1990. Pediatr Res 2013;74:50-72.

2. Lawn JE, Blencowe H, Oza S, et al.; Lancet Every Newborn Study Group. Every Newborn: progress, priorities, and potential beyond survival. Lancet 2014;384:189-205.

3. Rutherford MA, Pennock JM, Dubowitz LM. Cranial ultrasound and magnetic resonance imaging in hypoxic-ischaemic encephalopathy: a comparison with outcome. Dev Med Child Neurol 1994;36:813-25.

4. Cowan F, Rutherford M, GroenendaalF, etal. Origin and timing of brainlesions in term infants with neonatal encephalopathy. Lancet 2003;361:736-42.

5. Martinez-Biarge M, Diez-Sebastian J, Wusthoff CJ, Mercuri E, Cowan FM. Antepartum and intrapartum factors preceding neonatal hypoxic-ischemic encephalopathy. Pediatrics 2013;132:e952-9.

6. Kurinczuk JJ, White-Koning M, Badawi N. Epidemiology of neonatal encephalopathy and hypoxic-ischaemic encephalopathy. Early Hum Dev 2010;86:329-38.

7. Lawn JE, Lee AC, Kinney M, et al. Two million intrapartum-related stillbirths and neonatal deaths: where, why, and what can be done? Int J Gynaecol Obstet 2009;107:S5-18, S9.

8. Eklind S, Mallard C, Arvidsson P, Hagberg H. Lipopolysaccharide induces both a primary and a secondary phase of sensitization in the developing rat brain. Pediatr Res 2005;58:112-6.

9. Mallard C, Welin AK, Peebles D, Hagberg H, Kjellmer I. White matter injury following systemic endotoxemia or asphyxia in the fetal sheep. Neurochem Res 2003;28:215-23.

10. Soul JS, Robertson RL, Tzika AA, du Plessis AJ, Volpe JJ. Time course of changes in diffusion-weighted magnetic resonance imaging in a case of neonatal encephalopathy with defined onset and duration of hypoxicischemic insult. Pediatrics 2001;108:1211-4.

11. Thayyil S, Chandrasekaran M, Taylor A, et al. Cerebral magnetic resonance biomarkers in neonatal encephalopathy: a meta-analysis. Pediatrics 2010;125:e382-95.

12. Robertson NJ, Thayyil S, Cady EB, Raivich G. Magnetic resonance spectroscopy biomarkers in term perinatal asphyxial encephalopathy: from neuropathological correlates to future clinical applications. Curr Pediatr Rev 2014;10:37-47.

13. Rutherford MA, Pennock JM, Counsell SJ, et al. Abnormal magnetic resonance signal in the internal capsule predicts poor neurodevelopmental outcome in infants with hypoxic-ischemic encephalopathy. Pediatrics 1998;102(2 Pt 1):323-8. 


\section{Articles Tannetal.}

14. Wyatt JS, Edwards AD, Azzopardi D, Reynolds EO. Magnetic resonance and near infrared spectroscopy for investigation of perinatal hypoxicischaemic brain injury. Arch Dis Child 1989;64(7 Spec No):953-63.

15. Cotten CM, Shankaran S. Hypothermia for hypoxic-ischemic encephalopathy. Expert Rev Obstet Gynecol 2010;5:227-39.

16. Eken P, Jansen GH, Groenendaal F, Rademaker KJ, de Vries LS. Intracranial lesions in the fullterm infant with hypoxic ischaemic encephalopathy: ultrasound and autopsy correlation. Neuropediatrics 1994;25:301-7.

17. Leijser LM, Vein AA, Liauw L, Strauss T, Veen S, Wezel-Meijler Gv. Prediction of short-term neurological outcome in full-term neonates with hypoxic-ischaemic encephalopathy based on combined use of electroencephalogram and neuro-imaging. Neuropediatrics 2007;38:219-27.

18. Swarte R, Lequin M, Cherian P, Zecic A, van Goudoever J, Govaert P. Imaging patterns of brain injury in term-birth asphyxia. Acta Paediatr 2009;98:586-92.

19. Eken P, Toet MC, Groenendaal F, de Vries LS. Predictive value of early neuroimaging, pulsed Doppler and neurophysiology in full term infants with hypoxic-ischaemic encephalopathy. Arch Dis Child Fetal Neonatal Ed 1995;73:F75-80.

20. Boo NY, Chandran V, Zulfiqar MA, et al. Early cranial ultrasound changes as predictors of outcome during first year of life in term infants with perinatal asphyxia. J Paediatr Child Health 2000;36:363-9.

21. De Vries LS, Smet M, Goemans N, Wilms G, Devlieger H, Casaer P. Unilateral thalamic haemorrhage in the pre-term and full-term newborn. Neuropediatrics 1992;23:153-6.

22. Hagmann CF, Robertson NJ, Acolet D, et al. Cranial ultrasound findings in well newborn Ugandan infants. Arch Dis Child Fetal Neonatal Ed 2010;95:F338-44.

23. Lally PJ, Price DL, Pauliah SS, et al. Neonatal encephalopathic cerebral injury in South India assessed by perinatal magnetic resonance biomarkers and early childhood neurodevelopmental outcome. PLoS One 2014;9:e87874.

24. Iwata O, Iwata S, Thornton JS, et al. "Therapeutic time window" duration decreases with increasing severity of cerebral hypoxia-ischaemia under normothermia and delayed hypothermia in newborn piglets. Brain Res 2007;1154:173-80.

25. Barnette AR, Horbar JD, Soll RF, et al. Neuroimaging in the evaluation of neonatal encephalopathy. Pediatrics 2014;133:e1508-17.

26. Thompson CM, Puterman AS, Linley LL, et al. The value of a scoring system for hypoxic ischaemic encephalopathy in predicting neurodevelopmental outcome. Acta Paediatr 1997;86:757-61.

27. Sarnat HB, Sarnat MS. Neonatal encephalopathy following fetal distress. A clinical and electroencephalographic study. Arch Neurol 1976;33:696-705.

28. Tann CJ, Nkurunziza P, Nakakeeto M, et al. Prevalence of bloodstream pathogens is higher in neonatal encephalopathy cases vs. controls using a novel panel of real-time PCR assays. PLoS One 2014;9:e97259.

29. Hagmann CF, Robertson NJ, Acolet D, et al. Cerebral measurements made using cranial ultrasound in term Ugandan newborns. Early Hum Dev 2011;87:341-7.

30. Robertson NJ, Hagmann CF, Acolet D, et al. Pilot randomized trial of therapeutic hypothermia with serial cranial ultrasound and 18-22 month follow-up for neonatal encephalopathy in a low resource hospital setting in Uganda: study protocol. Trials 2011;12:138.

(i) This work is licensed under a Creative Commons Attribution 4.0 International License. The images or other third party material in this article are included in the article's Creative Commons license, unless indicated otherwise in the credit line; if the material is not included under the Creative Commons license, users will need to obtain permission from the license holder to reproduce the material. To view a copy of this license, visit http://creativecommons. org/licenses/by/4.0/

(C) CJTann et al. (2016) 